\title{
The efficacy of the semi-blind approach of transversus abdominis plane block on postoperative analgesia in patients undergoing inguinal hernia repair: a prospective randomized double-blind study
}

This article was published in the following Dove Press journal:

Local and Regional Anesthesia

17 January 2013

Number of times this article has been viewed

\author{
A Ebru Salman' \\ Fahri Yetișir ${ }^{2}$ \\ Banu Yürekli ${ }^{3}$ \\ Mustafa Aksoy' \\ Murat Yıldırım² \\ Mehmet Kılıç² \\ 'Anesthesiology and Reanimation \\ Department, ${ }^{2}$ General Surgery \\ Department, Atatürk Research and \\ Training Hospital, Ankara, Turkey; \\ ${ }^{3}$ Endocrinology Department, Bozyaka \\ Research and Training Hospital, Izmir, \\ Turkey
}

Purpose: In this prospective, randomized, double-blind study, our aim was to compare the analgesic efficacy of the semi-blind approach of transversus abdominis plane (TAP) block with a placebo block in patients undergoing unilateral inguinal hernia repair.

Methods: After receiving hospital ethical committee approval and informed patient consents, American Society of Anesthesiologists (ASA) I-III patients aged 18-80 were enrolled in the study. Standard anesthesia monitoring was applied to all patients. After premedication, spinal anesthesia was administered to all patients with $3.5 \mathrm{~mL}$ heavy bupivacaine at the $\mathrm{L}_{3}-\mathrm{L}_{4}$ subarachnoid space. Patients were randomly allocated into 2 groups. Group I $(n=32)$ received a placebo block with $20 \mathrm{~mL}$ saline, Group II $(\mathrm{n}=32)$ received semi-blind TAP block with $0.25 \%$ bupivacaine in $20 \mathrm{~mL}$ with a blunt regional anesthesia needle into the neurofascial plane via the lumbar triangle of Petit near the midaxillary line before fascial closure. At the end of the operation, intravenous (IV) dexketoprofen was given to all patients. The verbal analog scale (VAS) was recorded at 2, 4, 6, 12, and 24 hours postoperatively. Paracetamol IV was given to patients if their VAS score $>3$. A rescue analgesic of $0.05 \mathrm{mg} / \mathrm{kg}$ morphine IV was applied if VAS $>3$. Total analgesic consumption and morphine requirement in 24 hours were recorded.

Results: TAP block reduced VAS scores at all postoperative time points $(P<0.001)$. Postoperative analgesic and morphine requirement in 24 hours was significantly lower in group II $(P<0.01)$.

Conclusion: Semi-blind TAP block provided effective analgesia, reducing total 24-hour postoperative analgesic consumption and morphine requirement in patients undergoing elective unilateral inguinal hernia repair.

Keywords: pain, inguinal hernia, transversus abdominis plane

\section{Introduction}

Transversus abdominis plane (TAP) block is a novel technique that aims to improve postoperative pain control after abdominal surgery. ${ }^{1,2}$ It involves the block of the nerves of the anterior abdominal wall by the injection of a long-acting local anesthetic solution between the internal oblique and transversus abdominis muscles. ${ }^{3}$ It has been shown to be effective in several clinical settings, such as abdominoplasty, caesarian delivery, prostatectomy, and colorectal surgery. ${ }^{4-7}$
Correspondence: Fahri Yetișir General Surgery Department, Atatürk Research and Training Hospital, Mustafa Kemal mah. 2157. St.No:I I/8 Çankaya, Ankara, Turkey

Tel +90 5362974888

Email drfahriyetisir@hotmail.com 
Direct block of the afferent nerves of the abdominal wall, such as ilioinguinal and hypogastric nerve blocks, have been used to provide postoperative analgesia in patients undergoing open inguinal hernioraphy. ${ }^{8}$ TAP block is an alternative, simple to perform, and effective peripheral abdominal field block that blocks the lower intercostal (T7-T11), ilioinguinal, and hypogastric nerves. It is a modified technique in which the local anesthetic is injected in the neurovascular plane between the transversus abdominis muscle and internal oblique muscle of the anterior of the abdominal wall via the lumbar triangle of Petit. It is bounded posteriorly by the latissimus dorsi muscle and anteriorly by the external oblique and iliac crest forming the base of the triangle. ${ }^{9}$

In this study, we aimed to investigate the effect of a semi-blind approach of TAP block on postoperative verbal analog scale (VAS) scores and total analgesic and morphine consumption in the 24-hour postoperative period and compare it with the placebo block on patients undergoing elective inguinal hernia repair surgery under spinal anesthesia.

\section{Materials and methods}

After the hospital ethics committee approval and written informed patient consent, 64 American Society of
Anesthesiologists (ASA) physical status I-III patients aged 18-80 scheduled for elective primary unilateral open inguinal hernia repair were enrolled in a randomized, double-blind, controlled clinical trial. Only the patients with indirect hernias, fully reducible according to the Nyhus classification, ${ }^{10}$ were included in the study. Exclusion criteria were scrotal hernias, recurrent hernias, contraindication to spinal anesthesia, failure in spinal anesthesia, body mass index $(\mathrm{BMI})>35 \mathrm{~kg} / \mathrm{m}^{2}$, known allergy or contraindication to study drugs, chronic hepatic or renal failure, and preoperative opioid or nonsteroidal anti-inflammatory drug treatment for chronic pain (Figure 1).

Patients were allocated randomly by sealed, opaque envelopes - which were not opened until patient consent had been obtained - into groups according to a computergenerated sequence of random numbers. The patients, their anesthesiologists, investigators providing postoperative care, and the surgeon were blinded to group assignment. All patients were premedicated with midazolam at a dose of $0.02 \mathrm{mg} / \mathrm{kg}$ intravenous (IV) before surgery. Heart rate, noninvasive blood pressure, and peripheric $\mathrm{O}_{2}$ saturation were monitored during surgery in 15-minute intervals. Supplemental oxygen was administered to all patients via a nasal cannula. Patients were

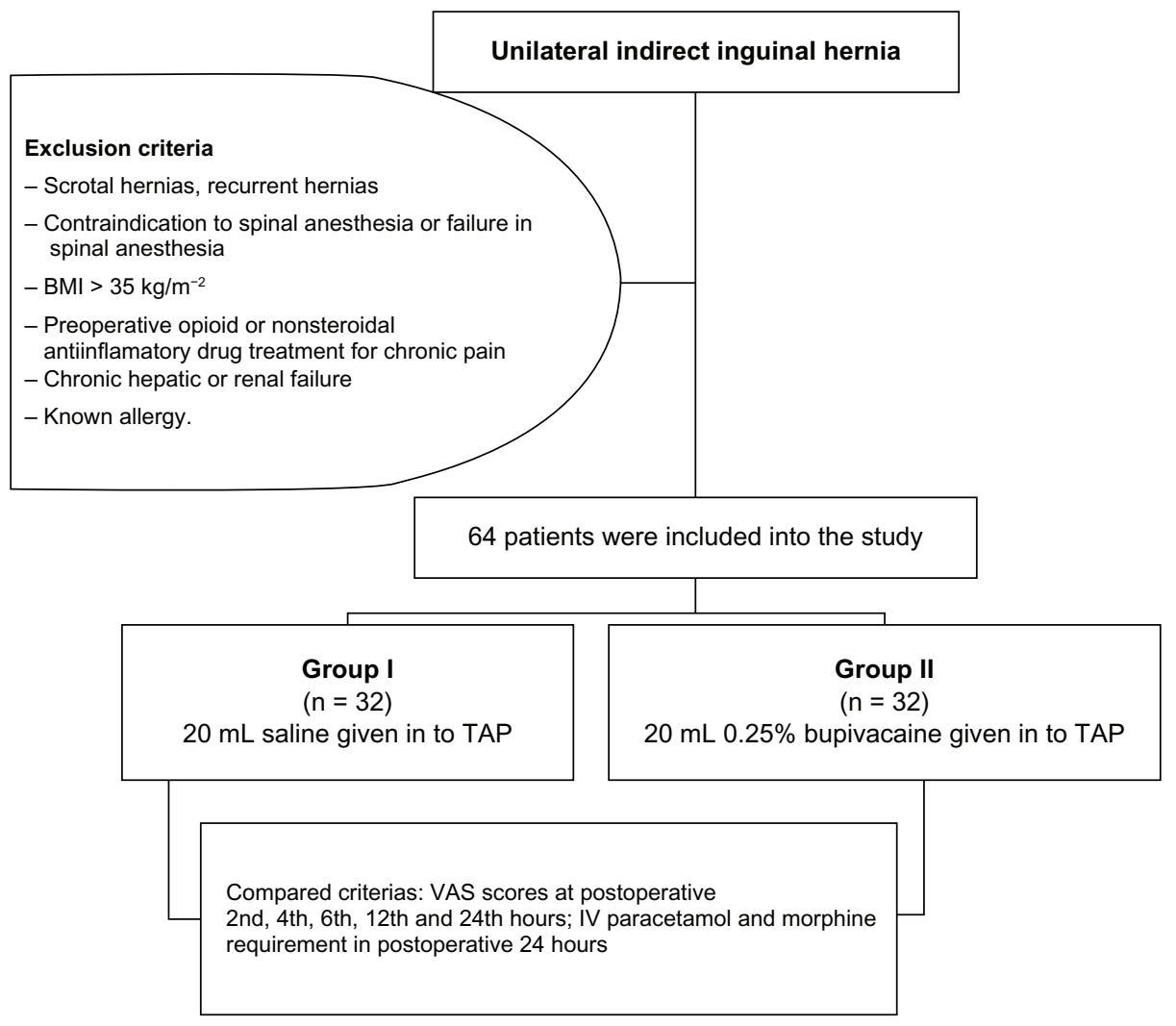

Figure I Flow chart of study.

Abbreviations: BMI, body mass index; IV, intravenous; TAP, transversus abdominis plane; VAS, verbal analog scale. 
placed in the lateral decubitus position. Spinal anesthesia was applied to all patients with $3.5 \mathrm{~mL}$ of heavy bupivacaine in the $\mathrm{L}_{3}-\mathrm{L}_{4}$ subarachnoid space. The surgery was begun after checking the level of block with the pin-prick test every 2 minutes, until the block remained at the same level three consecutive times. The patients were randomly assigned to group I (the patients who did not receive TAP block) or group II (the patients who did receive TAP block). All the patients were operated on by the same surgeon. Lichtenstein technique was used for inguinal hernia repair in both groups. A propylene mesh was placed to fit the floor of the inguinal canal and its apex was sutured to the pubic tubercle using a 3-0 prolene suture. The lower border of the mesh was attached to the free edge of the inguinal ligament after making an opening to accommodate the spermatic cord. The mesh was anchored to the conjoint tendon by interrupted sutures. At the end of the operation, after the mesh was placed, the iliac crest was palpated in order to check the insertion point of the needle; then the skin, which was marked before surgery by the anesthesiologist, was pierced with a blunt regional anesthesia needle (Plexufix; B Braun, Melsungen, Germany) at the level of the Petit triangle near the midaxillary line. The needle was advanced at right angles to the skin in a coronal plane, until resistance was encountered. This showed that the needle tip was at an external oblique muscle. The needle was gently advanced and the tip of the needle was gently controlled in the open surgical plane by the surgeon until it entered the plane between the internal and external oblique muscles. After passing through the internal oblique muscle and further advancement into the transversus abdominis fascial plane, the needle was carefully aspirated to exclude vascular puncture. $20 \mathrm{~mL}$ of $0.25 \%$ bupivacaine or the same volume of saline was injected in incremental doses through needle (Figure 2). All the blocks were performed by the same surgeon at the end of the surgery before fascial closure. Study solutions were prepared by an anesthesiologist who was not involved in performing the block or data collection.

Operation time, systolic arterial pressure, and diastolic and mean arterial pressures were recorded in 15 minutes intervals during operation. At the end of the operation, $50 \mathrm{mg}$ of IV dexketoprofen was injected into all patients. Pain assessments were scored for all patients at rest with coughing at 2, 4, 6, 12, and 24 hours after surgery by an anesthesiologist (independent observer) who did not know the group assignment. VAS (0: no pain to 10: worst imaginable pain) was used for pain assessment, for which all patients received instructions prior to surgery. Patients were given IV paracetamol if VAS $>3$, maximally 4 times a day at 6-hour intervals. As a rescue analgesic, morphine IV 0.05 $\mathrm{mg} / \mathrm{kg}$ was applied to patients if their VAS score was $>3$, if paracetamol had been administered less than 6 hours before. Morphine injection was repeated up to $15 \mathrm{mg}$ in 4 hours. At

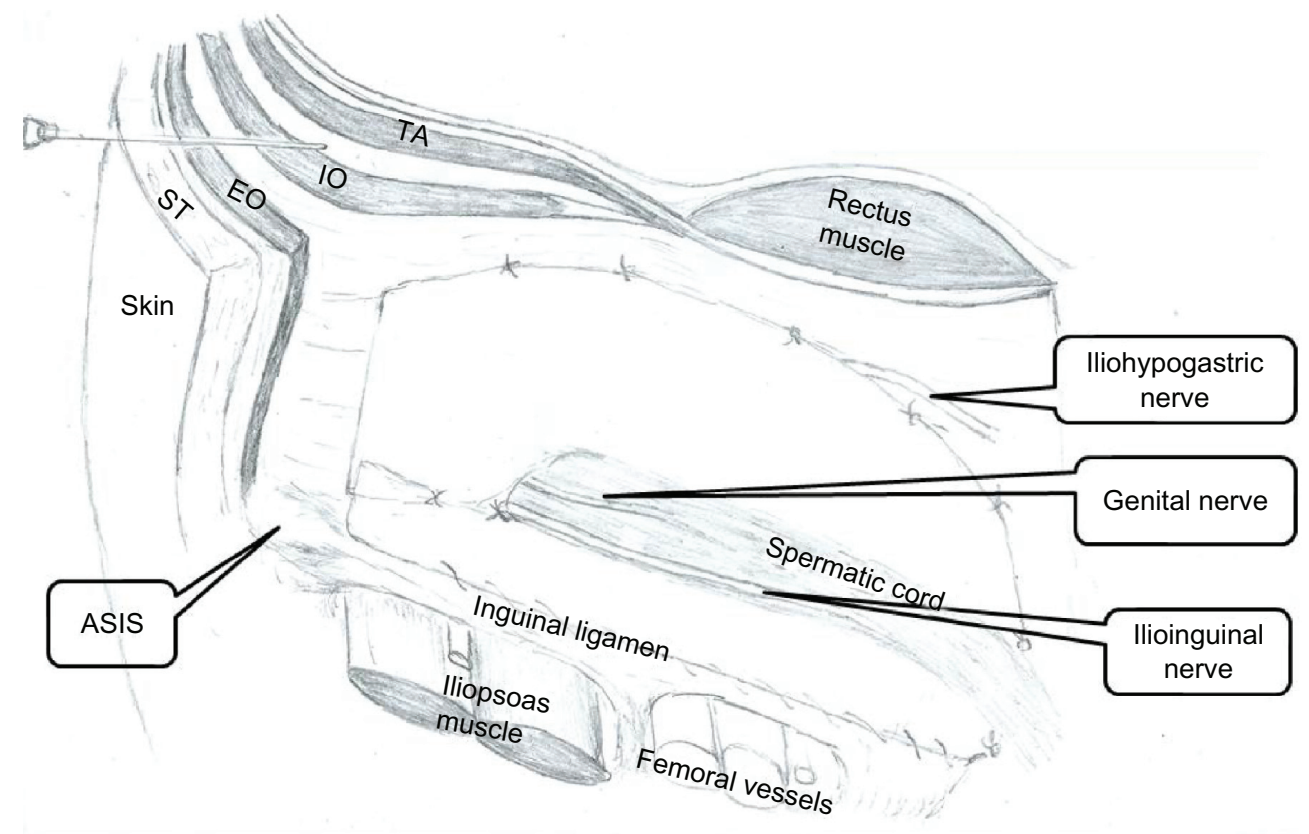

Figure 2 Insertion of a blunt needle penetrating the fascia of the external and internal oblique muscles before entering the transversus abdominis fascial plane. Abbreviations: ASIS, anterior superior iliac spine; EO, external oblique muscle; IO, internal oblique muscle; ST, subcutaneous tissue; TA, transversus abdominis muscle. 
any time, if pain relief was inadequate, an alternative opioid (tramadol) IV at a dose of $1 \mathrm{mg} / \mathrm{kg}$ was used. Total analgesic consumption and morphine requirement in the 24-hour postoperative period were also recorded. Any complications related to interventions were also noted.

\section{Statistical analysis}

Data analysis was performed by using Statistical Package for Social Sciences (SPSS) version 17.0 software (IBM Corporation, Armonk, NY). Data were expressed as mean \pm standard deviation. Kolmogorov Smirnov test was used to identify the distribution of variables. Nonparametric statistical methods were used for the heterogeneous variables. The Mann-Whitney $U$ test was used for nonparametric variables. A $P$-value less than 0.05 was considered statistically significant.

\section{Sample size estimation}

A power analysis based on previously published data ${ }^{11}$ suggested that a total sample size of 50 (25 per group) was required to detect at least $30 \%$ of the difference in VAS scores for pain between study and control groups with a power of $80 \%$ at the $5 \%$ significance level. We also ran a pilot study ( 5 patients per group) for power analysis. The difference of $50 \%$ was taken from our pilot study. Sample size estimation was performed by using NCSS and PASS 2000 software (NCSS LLC, Kaysville, UH).

\section{Results}

Demographic variables were similar between the groups (Table 1). The differences in mean blood pressure were found to be similar between groups. Also, there were no statistically significant differences in mean blood pressure among measurement times within both group I and II.

The differences in heart rate were found to be similar between groups. Also, there were no statistically significant differences in heart rate among measurement times within both group I and II. The differences in oxygen saturation were found to be similar between groups.

VAS scores were lower in group II at all postoperative time points $(P<0.001$, Figure 3$)$. Demand for analgesic additional

Table I Demographic and clinical characteristics

\begin{tabular}{llll}
\hline Variable & Group I & Group II & P-value \\
\hline Age, years & $54.4 \pm 15.7$ & $54.0 \pm 11.8$ & 0.930 \\
Sex (F/M), n & $4 / 28$ & $2 / 30$ & 1.000 \\
ASA (I/II/III), n & $16 / I 5 / I$ & $16 / 10 / 6$ & 0.669 \\
BMI & $25.5 \pm 5.4$ & $25.9 \pm 5 . I$ & 0.978 \\
\hline
\end{tabular}

Abbreviations: F, female; M, male; ASA, American Society of Anesthesiologists; BMI, body mass index. to IV dexketoprofen at $4(P<0.001), 6(P<0.001), 12$ $(P<0.001)$, and 24 hours $(P=0.017)$ postoperatively was statistically significant (Figure 4). Morphine requirement in the 24-hour postoperative period was higher in group I (Figure 5). This difference was statistically significant $(P<0.001)$. We did not observe any complications associated with interventions.

\section{Discussion}

We observed that the semi-blind approach of TAP block provided effective postoperative analgesia after elective inguinal hernia repair under spinal anesthesia and reduced total analgesic consumption and morphine requirement in the 24-hour postoperative period.

Various studies have demonstrated that TAP block provides effective analgesia and decreases postoperative morphine requirement after caesarian delivery, abdominal hysterectomy, retro-pubic prostatectomy, colorectal surgery, inguinal hernia repair, and abdominal surgery. ${ }^{5-7,12-16}$ Aveline et al recently showed that ultrasound guided TAP block provided better pain relief and reduced the opioid requirement when compared with conventional iliohypogastric nerve blocks. ${ }^{15}$

On the other hand, Griffiths et al showed that bilateral ultrasound guided TAP block failed to show any additional benefit to multimodal analgesia in patients undergoing midline laparotomy: ${ }^{17}$ this finding contrasts with recent literature. ${ }^{4-7}$ The population of the Griffith et al study was heterogeneous in terms of age, BMI, and height of surgical incision. On the other hand, an abdominal field block (eg, TAP block) can only provide analgesia for the parietal component of postoperative pain of major gynecologic surgery which consists of procedures ranging from relatively minor procedures to formal staging laparotomy. ${ }^{17}$ In our study, none of our patients was morbidly obese and surgical insult was consistent and produced small to moderate surgical trauma. This may be one of the reasons why we observed such a big difference between groups in postoperative VAS scores and total analgesic consumption and morphine requirement in the 24-hour postoperative period.

Several approaches have been described for performing TAP block - the classic posterior blind approach and ultrasound-guided approaches. The blind technique was initially described by Rafi. ${ }^{18}$ It involves injection of local anesthetic solution into the TAP using the double pop of piercing the fascial planes via the iliolumbar triangle of Petit.

In a recent approach, often called the subcostal approach, local anesthetic is injected into the same anatomic plane using an insertion point near the xiphoid process. This approach 


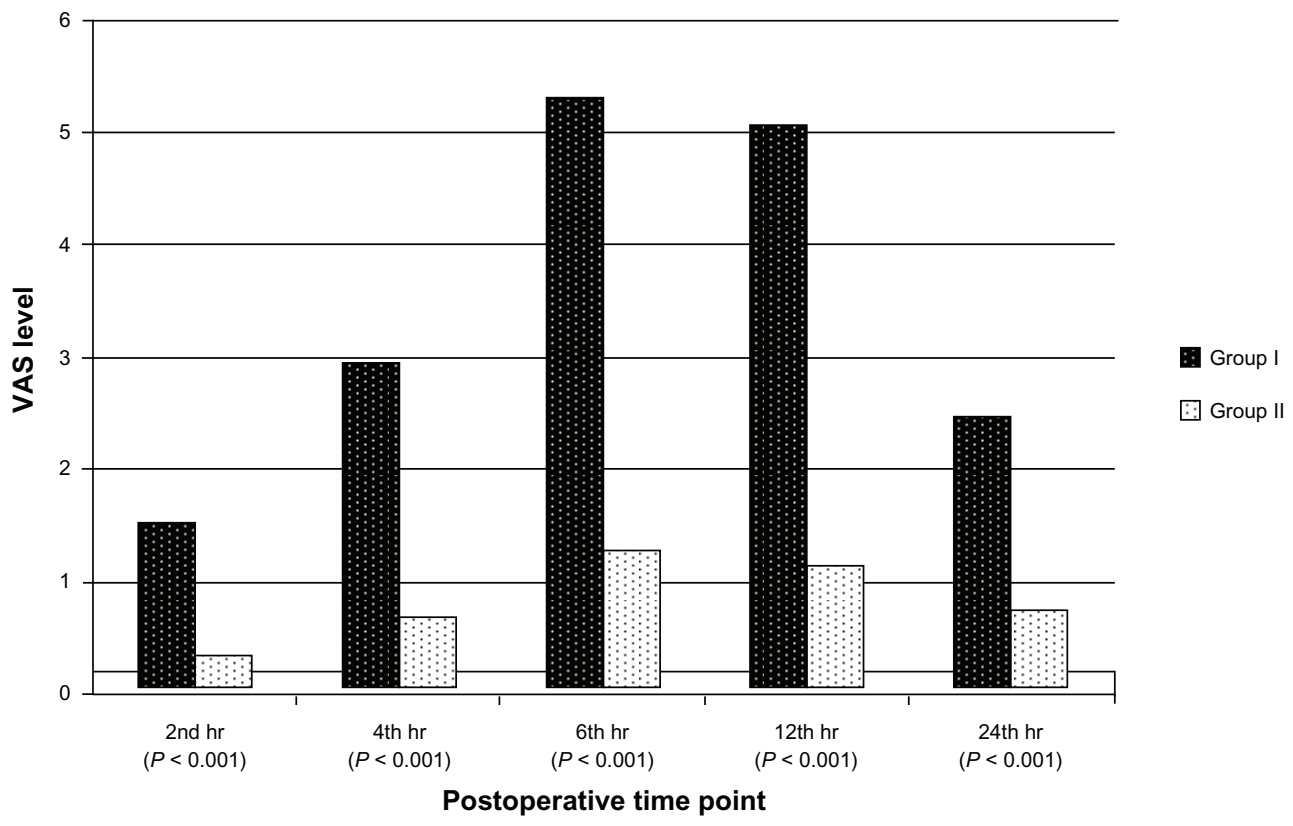

Figure 3 VAS levels by postoperative measurement time.

Abbreviations: VAS, verbal analog scale; hr, hour.

may offer superior analgesia for abdominal operations above the umbilicus. ${ }^{17}$ On the other hand, Jankovic et al showed in their cadaveric study that the position of the lumbar triangle of Petit varies largely and its size is small, and that the nerves to be blocked had not entered TAP in the specimens at the point of the lumbar triangle of Petit. ${ }^{19,20}$ This makes the performance of TAP block less convenient and more difficult in supine patients. It was also demonstrated that, at the midaxillary line, all the nerves were within the transversus abdominis plane. We performed TAP block in this study at the level of the Petit triangle near the midaxillary line, marking the insertion point of the needle before the surgery and controlling the position of the needle and spread of the local anesthetic in the open surgical plane in our semi-blind approach of TAP block.

Our study has several limitations. First of all, we did not assess pain scores and analgesic consumption after 24 hours because of our ward conditions. However, TAP block has been shown to produce effective analgesia for 48 hours postoperatively. ${ }^{6}$ We did not assess pain on movement but only assessed the pain at rest with coughing. TAP block reduced postoperative opioid use, although

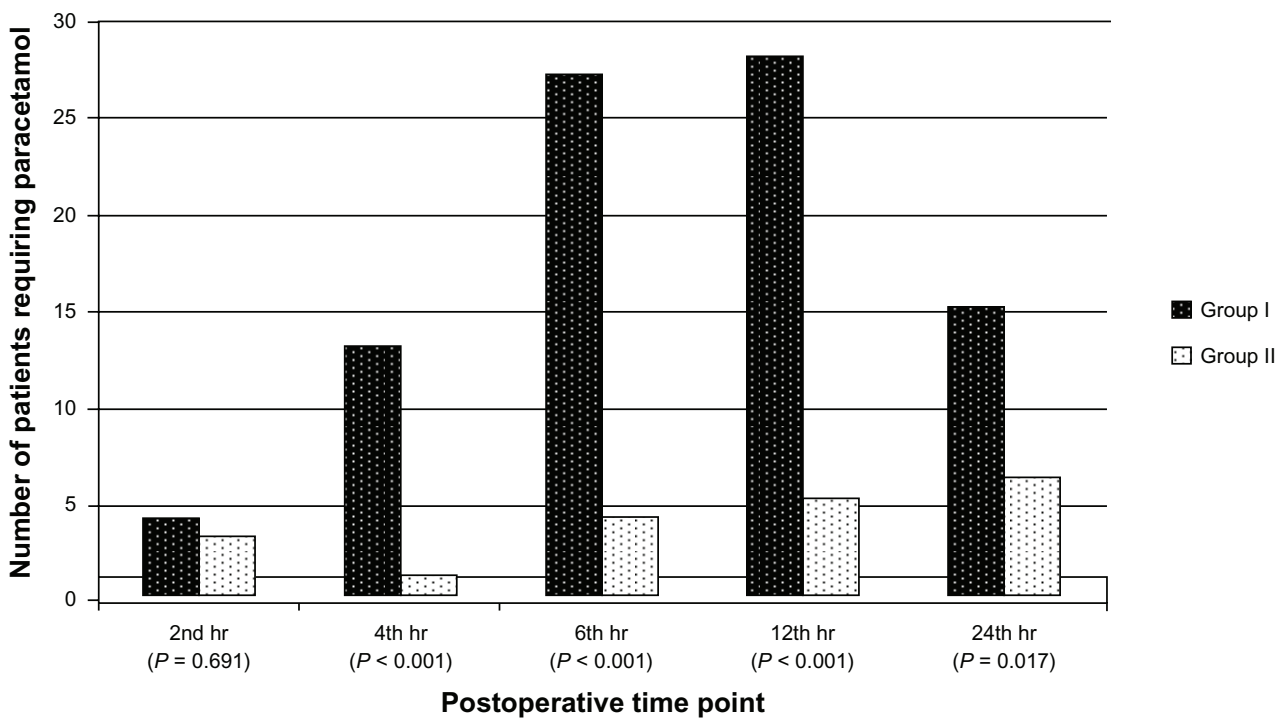

Figure 4 Demand for IV paracetamol by postoperative measurement time.

Abbreviations: IV, intravenous; hr, hour. 


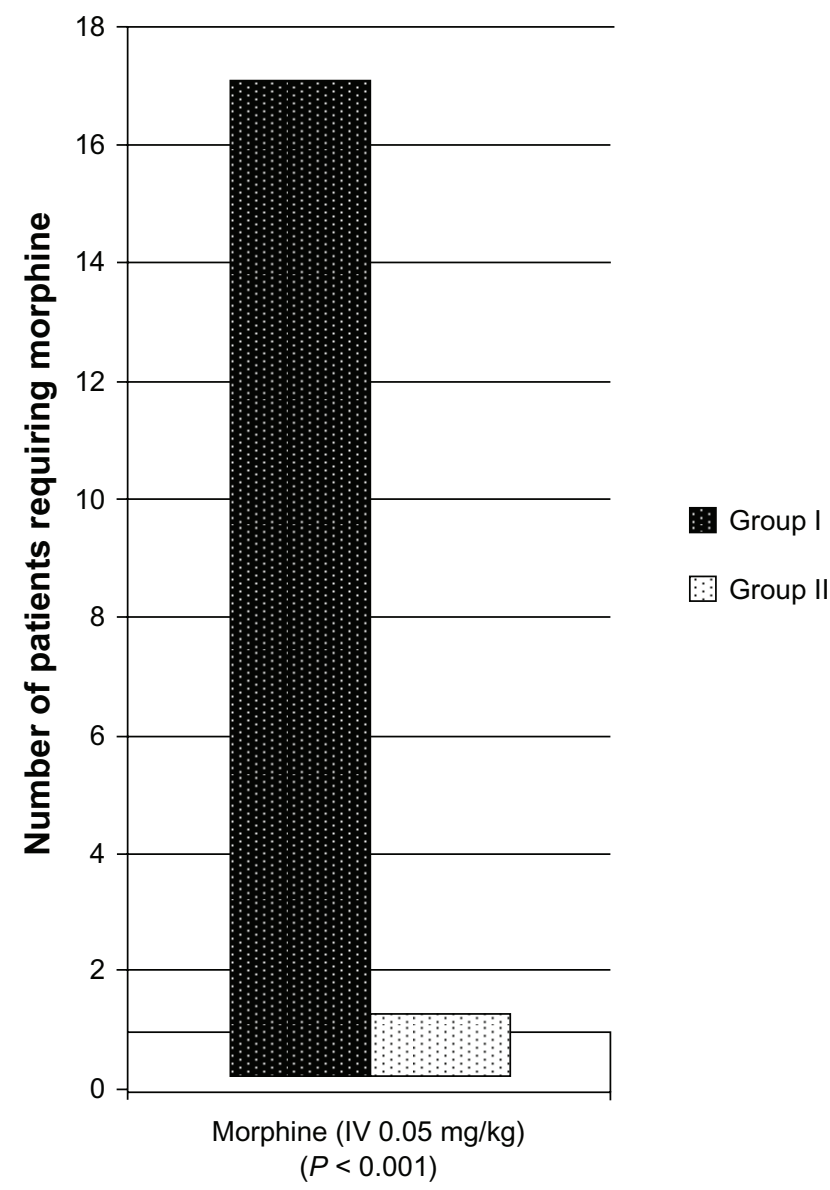

Figure 5 The frequency of total morphine required 24 hours postoperatively. Abbreviation: IV, intravenous.

we have no data on postoperative nausea and other opioid dependent side effects. Our findings may not be generalized to obese patients with different surgical subtypes, since our study was conducted on patients undergoing a specific type of surgery with moderate surgical trauma. Measurement of sensory blockade was not performed in the postoperative period so that investigators could be adequately blinded.

Some complications like puncture of intraperitoneal viscera and liver have been documented after a blind TAP block. ${ }^{21,23}$ McDermott et al demonstrated in their study that that the needle tip and local anesthetic spread were in the correct plane only in 17 (23\%) of 72 injections in 36 patients. ${ }^{23}$ In the remaining 55 patients the needle tip was in the subcutaneous tissue in one patient, the external oblique muscle in one patient, between the external and internal oblique muscles in five patients, the internal oblique muscle in 26 patients, the transversus abdominis muscle in nine patients, and the peritoneum in 13 patients. TAP block was performed bilaterally using the standard landmark based blind technique and ultrasonography was used to detect the needle position and local anesthetic spread in that study. The use of ultrasound may reduce such complications; we did not observe any complications in our patients, since we used the semi-blind technique. This might be caused by the fact that TAP block was performed by advancing the needle carefully, with the tip of the advancing needle controlled by the finger of the surgeon in the open surgical plane. Hence, the analgesic effect of TAP block may partly depend on the increase in the serum concentration of the local anesthetic. TAP block may cause systemic toxicity of local anesthetic, ${ }^{24}$ therefore, we used a relatively diluted amount of bupivacaine in the present study.

\section{Conclusion}

In conclusion, it was shown that semi-blind TAP block may be an effective way of providing analgesia in patients undergoing elective open inguinal hernia repair under spinal anesthesia.

\section{Disclosure}

The authors report no conflicts of interest in this work.

\section{References}

1. Siddiqui MR, Sajid MS, Uncles DR, Cheek L, Baig MK. A metaanalysis on the clinical effectiveness of transversus abdominis plane block. J Clin Anesth. 2011;23(1):7-14.

2. Petersen PL, Mathiesen O, Torup H, Dahl JB. The transversus abdominis plane block: a valuable option for postoperative analgesia? A topical review. Acta Anaesthesiol Scand. 2010;54(5):529-535.

3. Muhtkar K, Singh S. Transversus abdominis plane block for laparoscopic surrgery. Br J Anaesth. 2009;102(1):143-144.

4. Araco A, Pooney J, Araco F, Gravente G. Transversus abdominis plane block reduces the analgesic requirements after abdominoplasty with flank liposuction. Ann Plast Surg. 2010;65(4):385-388.

5. McDonell JG, Curley G, Carney J, et al. The analgesic efficacy of transversus abdominis plane block after cesarean delivery: a randomized controlled trial. Anesth Analg. 2008;106(1):186-191.

6. O'Donnell BD, McDonnell JG, McShane AJ. The transversus abdominis plane (TAP) block in open retropubic prostatectomy. Reg Anesth Pain Med. 2006;31(1):91.

7. Conaghan P, Maxwell-Armstrong C, Bedforth N, et al. Efficacy of transversus abdominis plane blocks in laparoscopic colorectal resections. Surg Endosc. 2010;24(10):2480-2484.

8. Dierking GW, Dahl JB, Kanstrup J, Dahl A, Kehlet H. Effect of pre- vs postoperative inguinal field block on postoperative pain after herniorrhaphy. Br J Anaesth. 1992;68(4):344-348.

9. Netter FH. Abdomen posterolateral abdominal wall. In: Netter FH, editor. Atlas of Human Anatomy Summit. New Jersey: The Ciba-Geigy Corporation; 1989:230-240.

10. Nyhus LM, Klein MS, Rogers FB. Inguinal hernia. Curr Probl Surg. 1991;28(6):401-450.

11. Song D, Greilich NB, White PF, Watcha MF, Tongier WK. Recovery profiles and costs of anesthesia for outpatient unilateral inguinal herniorrhaphy. Anesth Analg. 2000;91(4):876-881.

12. Belavy D, Cowlishhaw PJ, Howes M, Phillips F. Ultrasound-guided transversus abdominis plane block for analgesia after Caesarean delivery. Br J Anaesth. 2009;103(5):726-730. 
13. Carney J, McDonell JG, Ochana A, Bhinder R, Laffey JG. The transversus abdominis plane block provides effective postoperative analgesia in patients undergoing total abdominal hysterectomy. Anesth Analg. 2008;107(6):2056-2060.

14. Bharti N, Kumar P, Bala I, Gupta V. The efficacy of a novel approach to transversus abdominis plane block for postoperative analgesia after colorectal surgery. Anesth Analg. 2011;112(6):1504-1508.

15. Aveline C, Le Hetet H, Le Roux A, et al. Comparison between ultrasound-guided transversus abdominis plane and conventional ilioinguinal/iliohypogastric nerve blocks for day-case open inguinal hernia repair. Br J Anaesth. 2011;106(3):380-386.

16. McDonnell JG, O’Donnell B, Curley G, Heffernan A, Power C, Laffey JG. The analgesic efficacy of transversus abdominis plane block after abdominal surgery: a prospective randomized controlled trial. Anesth Analg. 2007;104(1):193-197.

17. Griffiths JD, Middle VJ, Baron FA, Grant SJ, Popham PA, Royse CF. Transversus abdominis plane block does not provide additional benefit to multimodal analgesia in gynecological cancer surgery. Anesth Analg 2010;111(3):797-801.
18. Rafi AN. Abdominal field block: a new approach via the lumbar triangle. Anaesthesia. 2001;56(10):1024-1026.

19. Jankovic ZB, du Feu FM, McConnell P. An anatomical study of the transversus abdominis plane block: location of the lumbar triangle of Petit and adjacent nerves. Anesth Analg. 2009;109(3):981-985.

20. Rozen WM, Tran TM, Ashton MW, Barrington MJ, Ivanusic JJ, Taylor GI. Refining the course of the thoracolumbar nerves: a new understanding of the innervation of the anterior abdominal wall. Clin Anat. 2008;21(4):325-333.

21. Jankovic Z, Ahmad N, Ravishankar N, Archer F. Transversus abdominis plane block: how safe is it? Anesth Analg. 2008;107(5):1758-1759.

22. Faroog M, Carey M. A case of liver trauma with a blunt regional anesthesia needle while performing transversus abdominis plane block. Reg Anesth Pain Med. 2008;33(3):274-275.

23. McDermott G, Korba E, Mata U, et al. Should we stop doing blind transversus abdominis plane blocks? Br J Anaesth. 2012;108(3):499-502.

24. Kato N, Fujiwara Y, Harato M, et al. Serum concentrations of lidocaine after transversus abdominis plane block. J Anesth. 2009; 23(2):298-300.
Local and Regional Anesthesia

\section{Publish your work in this journal}

Local and Regional Anesthesia is an international, peer-reviewed, open access journal publishing on the development, pharmacology, delivery and targeting and clinical use of local and regional anesthetics and analgesics. The journal welcomes submitted papers covering original research, basic science, clinical studies, reviews \& evaluations,

\section{Dovepress}

guidelines, expert opinion and commentary, case reports and extended reports. The manuscript management system is completely online and includes a very quick and fair peer-review system, which is all easy to use. Visit http://www.dovepress.com/testimonials.php to read real quotes from published authors. 